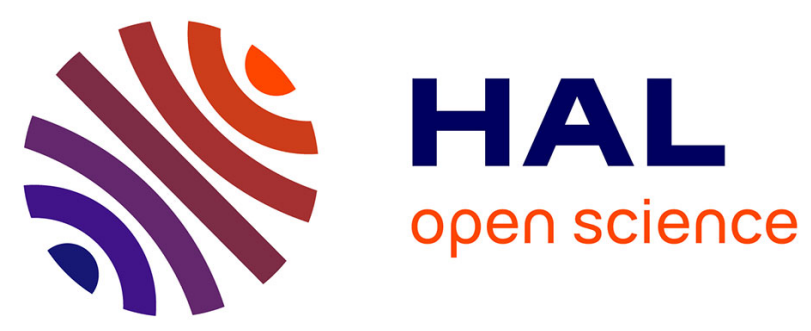

\title{
Defects characterization in thin films photovoltaics materials by correlated high-frequency modulated and time resolved photoluminescence: An application to $\mathrm{Cu}(\mathrm{In}, \mathrm{Ga}) \mathrm{Se} 2$
}

Baptiste Bérenguier, Nicolas Barreau, Alexandre Jaffré, Daniel Ory, Jean-Francois Guillemoles, Jean-Paul Kleider, Laurent Lombez

\section{To cite this version:}

Baptiste Bérenguier, Nicolas Barreau, Alexandre Jaffré, Daniel Ory, Jean-Francois Guillemoles, et al.. Defects characterization in thin films photovoltaics materials by correlated high-frequency modulated and time resolved photoluminescence: An application to $\mathrm{Cu}(\mathrm{In}, \mathrm{Ga}) \mathrm{Se} 2$. Thin Solid Films, 2019, 669, pp.520-524. 10.1016/j.tsf.2018.11.030 . hal-02057754

\author{
HAL Id: hal-02057754 \\ https://hal.science/hal-02057754
}

Submitted on 29 Oct 2019

HAL is a multi-disciplinary open access archive for the deposit and dissemination of scientific research documents, whether they are published or not. The documents may come from teaching and research institutions in France or abroad, or from public or private research centers.
L'archive ouverte pluridisciplinaire HAL, est destinée au dépôt et à la diffusion de documents scientifiques de niveau recherche, publiés ou non, émanant des établissements d'enseignement et de recherche français ou étrangers, des laboratoires publics ou privés. 


\section{Accepted Manuscript}

Defects characterization in thin films photovoltaics materials by correlated high-frequency modulated and time resolved photoluminescence: An application to $\mathrm{Cu}(\mathrm{In}, \mathrm{Ga}) \mathrm{Se} 2$

Baptiste Bérenguier, Nicolas Barreau, Alexandre Jaffre, Daniel Ory, Jean François Guillemoles, Jean-Paul Kleider, Laurent Lombez

PII:

S0040-6090(18)30771-5

DOI: https://doi.org/10.1016/j.tsf.2018.11.030

Reference: TSF 37004

To appear in:

Thin Solid Films

Received date: 5 July 2018

Revised date: 13 November 2018

Accepted date: 15 November 2018

Please cite this article as: Baptiste Bérenguier, Nicolas Barreau, Alexandre Jaffre, Daniel Ory, Jean François Guillemoles, Jean-Paul Kleider, Laurent Lombez , Defects characterization in thin films photovoltaics materials by correlated high-frequency modulated and time resolved photoluminescence: An application to $\mathrm{Cu}(\mathrm{In}, \mathrm{Ga}) \mathrm{Se} 2$. Tsf (2018), https://doi.org/10.1016/j.tsf.2018.11.030

This is a PDF file of an unedited manuscript that has been accepted for publication. As a service to our customers we are providing this early version of the manuscript. The manuscript will undergo copyediting, typesetting, and review of the resulting proof before it is published in its final form. Please note that during the production process errors may be discovered which could affect the content, and all legal disclaimers that apply to the journal pertain. 


\title{
Defects characterization in thin films photovoltaics materials by correlated high-frequency modulated and time resolved photoluminescence: an application to $\mathrm{Cu}(\mathrm{In}, \mathrm{Ga}) \mathrm{Se}_{2}$
}

\author{
Baptiste Bérenguier ${ }^{\mathrm{a}, \mathrm{b},{ }^{,}}$, Nicolas Barreau ${ }^{c}$, Alexandre Jaffre ${ }^{\mathrm{a}, \mathrm{d}}$, Daniel Ory ${ }^{\mathrm{a}, \mathrm{e}}$, Jean François \\ Guillemoles $^{\mathrm{a}, \mathrm{b}}$, Jean-Paul Kleider ${ }^{\mathrm{a}, \mathrm{d}}$, Laurent Lombez ${ }^{\mathrm{a}, \mathrm{b}}$
}

a) Institut Photovoltaïque d'Ile de France, 30 Route Départementale 12891120 Palaiseau, France

b) CNRS, UMR IPVF 9006, Institut Photovoltaïque d'Ile de France, 30 Route Départementale 128 91120 Palaiseau, France

c) Institut des Matériaux Jean Rouxel (IMN), Université de Nantes, CNRS, 2 rue de la Houssinière, 44322 Nantes, France

d) GeePs, UMR 8507-CNRS, CentraleSupélec, Univ. Paris-Sud, Université Paris-Saclay, Sorbonne Université, 11 rue Joliot-Curie, F-91192 Gif-surYvetteCedex, FRANCE

e) EDF R\&D, 30 Route Départementale 12891120 Palaiseau, France

Abstract. We develop a contactless method based on photoluminescence measurements in the modulated mode: the high-frequency modulated photoluminescence. The high frequency domain allows accessing to carrier dynamics in the nanosecond time scale which is typical for thin films materials. To illustrate the experimental method, we analyze $\mathrm{Cu}(\mathrm{In}, \mathrm{Ga}) \mathrm{Se}_{2}$ photovoltaic absorbers where recombination mechanisms in the bulk, surface and grain boundaries are not completely understood. We correlate the data with classical time resolved photoluminescence. We show that the combination of the two methods allows, with the help of one dimensional simulations, an estimation of carrier traps and recombination centers parameters in thin films samples.

Keywords: Modulated Photoluminescence, Transient Photoluminescence, Time Resolved Photoluminescence, Carrier Traps, Copper Indium Gallium Selenide

\section{Introduction}

Modulated Photoluminescence (MPL) is well-known in the silicon community to probe carrier lifetime and investigate the recombination mechanisms at a given working point [1-3]. However, the application of this experimental method on thin films materials is challenging as the carrier dynamics is usually in the nanosecond regime. Time Resolved Photoluminescence (TRPL) is, for its part, a wellestablished technique for probing fast recombination mechanisms in thin film semiconductors. However, the temporal decays might be difficult to interpret as they can strongly deviate from single exponential behavior associated with a simple notion of lifetime [4-6]. In fact several mechanisms play a role in the temporal decay of photoluminescence signals, including bulk, interfaces, and grain boundaries recombination, carrier trapping, and transport (thermionic emission at the heterojunction, diffusion and drift)... This is for instance the case in $\mathrm{Cu}(\mathrm{In}, \mathrm{Ga}) \mathrm{Se}_{2}$ (CIGS) materials, a promising thin films photovoltaic absorber we investigate in this study to illustrate the method we propose.

*Corresponding author: Institut Photovoltaïque d'Ile de France, 30 Route Départementale 12891120 Palaiseau, France. E-mail address: baptiste.berenguier@gmail.com 
In order to distinguish the influence of each phenomenon, one can perform a parametric study over one or several physical parameters that affect the studied kinetics. These are typically carrier concentration (and its modulation in time/space) and temperature. The illumination intensity is the easiest way to change the carrier density, impacting all the above mentioned phenomena [7,8], it can moreover be easily modulated. Voltage biasing allows to change minority carrier concentration and to control the space charge region width of the CIGS cells but it imposes to work on contacted junctions $[8,9]$ and implies electroluminescence in case of forward biasing. Temperature modification can influence recombination but also transport $[6,7]$. In this study, we explore the possibility to change the illumination temporal variations by using sinusoidal illumination at high frequencies, i.e. High Frequency Modulated Photoluminescence (HF-MPL).

While ultra-short laser pulses are used in TRPL experiments to record fast mechanisms (transport and recombination), the use of a slower illumination variation allows probing carrier dynamics in a way closer to the real excitation conditions and also to focus on slow mechanisms such as carrier detrapping. A trial has already been performed successfully by using modulated electroluminescence (MEL) on CIGS finished cells for trap detection [10], but on an opposite way, the maximum modulation frequency was limited at $200 \mathrm{kHz}$ which might not be sufficient to probe fast carriers' dynamics as it happens in thin films materials with direct bandgap. Based on previous experiences in modulated techniques $[3,11]$ we developed a HF-MPL setup extending the frequency range up to $10 \mathrm{MHz}$.

Furthermore, the mathematical background for data treatment of modulated luminescence [10] usually comes from the field of silicon crystalline wafers using the notion of effective lifetime. This is unclear in case of trapping events. In this work, we used a one dimensional time-dependent drift diffusion model to fit our results, which include sall the above cited mechanisms (with the exception of rigorous grain boundaries modelization because it is a three dimensional effect)

Finally, we performed HF-MPL and TRPL experiments on CIGS samples and compared the recorded data. Trap parameters were extracted by data fitting from both experimental methods.

\section{Experimental}

\subsection{Setup.}

The setup is sketched in Figure 1.a. The light sources are a $532 \mathrm{~nm}$-wavelength intensity modulated laser and a $640 \mathrm{~nm}$ pulsed laser with 80 ps pulse duration (full width at half maximum), respectively. The modulation is based on sinusoidal variations of the light intensity around a given working point. In this study the ratio between modulated and continuous part of the modulated light is close to unity in order to compare the results with TRPL and validate the approach. Note that the MPL also allows measuring small PL variations around a given working point (small signal mode). The light is sent to the sample via an injection fiber with $50 \mu \mathrm{m}$ core. A lens (one-inch focal length) is placed at the output of the fiber allows for focusing the laser beam. Note that the latter is not collimated to widen the excitation spot and make it much larger than the carrier diffusion length. A semi-reflective plate is then used. Samples are excited trough a microscope objective (numerical aperture 0.75 ) and the beam diameter was measured to be $60 \mu \mathrm{m}$ on the sample. The photoluminescence signal (PL) is then collected. To detect the signal with an optimal sensitivity we used a Single Photon Avalanche Detector. Then a Picoharp 300 Time Correlated Single Photon Counter (TCSPC) and a LabVIEW software allow for reconstructing one decay in TRPL mode or one period of the modulated signal (See Figure 1.b). In such configuration we obtain a unique experimental setup that allows to record fast modulated signal with a high detection sensitivity. In classical modulated experiments when one 
uses a fast detector, the sensitivity is often strongly degraded (compromise between speed and gain). Once the modulated signal is recorded, we can extract the phase and amplitude of the first harmonic. In the case of silicon, the fitting of the phase or the amplitude versus modulation frequency allows the determination of the effective carrier lifetime [2]. The phase is often preferred because it is closely related to the lifetime and less dependent to noise or signal distortions.

\subsection{Samples}

CIGS samples were fabricated at the Institut des Matériaux Jean Rouxel (University of Nantes). In order to simplify the data analysis, samples were grown without gallium gradient in a one stage coevaporation process. The absence of gradient was verified by Glow Discharge Optical Emission Spectroscopy analysis. The ratio $[\mathrm{Ga}] /([\mathrm{Ga}]+[\mathrm{In}])$ was measured to be $25 \%$. External Quantum Efficiency curves as well as PL spectra are compatible with a $1.15 \mathrm{eV}$ band gap. The sample was cut in three parts, one was kept as grown, one was covered by CdS, one was finished as a solar cell through the depositions of $\mathrm{CdS}, \mathrm{ZnO}$ and ohmic contacts. The efficiency was measured on the third part at $12.5 \%$ with a $588 \mathrm{mV}$ open circuit voltage, $31 \mathrm{~mA} . \mathrm{cm}^{-2}$ short circuit current density and a $69 \%$ fill factor.

\section{Simulation tool}

A one dimensional drift diffusion simulation code was developed functioning under Matlab as well as Octave environment [12] and allowing us to fit the HF-MPL/TRPL data. The code uses a spatial discretization of the drift diffusion equations as presented in [13] in combination with differential algebraic equations solvers for the temporal part $[14,15]$. This approach has been proved to be efficient, see for example [16]. The code also includes the following physical effects: radiative, Auger, Shockley Read Hall (SRH), surface and back contact recombinations, carrier trapping on defects via rate equations [6,17], thermionic emission at interfaces [18] and trap assisted tunneling [19,20]. The outputs provide the carriers densities profiles $n(x, t)$ and $p(x, t)$, as well as the electric potential for a given semiconductor stack with respect to depth and time. Light excitation can follow arbitrary time profiles, the absorption profile being given by Beer-Lambert approximation. Then the photoluminescence is approximated as the depth integration of the $n \times p$ product, providing the photoluminescence relativeintensity as a function of time $I_{P L}(t)$. In case of sinusoidal excitation, the phase and amplitude of $I_{P L}(t)$ first harmonic are calculated using usual Fourier series formulas.

The fitting procedure solves the drift-diffusion equations on several successive periods until stabilization of the solution. For each data set, the fit was performed simultaneously on all the experimental curves, corresponding to several excitation intensities. The fit used least square trust region reflective algorithm. With such method, we believe that the extracted parameters represent reliable material properties for different illumination conditions.

\section{Results and discussion}

\subsection{Results for the as grown absorber}

Figure 2 presents the HF-MPL phases as well as the TRPL decays measurements made on the as grown sample. In the HF-MPL experiment the continuous part of the illumination was varied from 1.3 $10^{19}$ photons $/ \mathrm{cm}^{2} / \mathrm{s}$ to $1.310^{21}$ photons $/ \mathrm{cm}^{2} / \mathrm{s}$ with a $\sqrt{10}$ multiplicative step which corresponds roughly to [130-13000] suns. In our case, the phase curves can be sorted into two distinct groups. The three low power curves present a distinct and asymmetric peak with a maximum at $250 \mathrm{kHz}(\mathrm{V}$ shape). This peak seems to disappear at higher illumination intensity and a monotonous variation is observed. In the TRPL decays, the illumination power was varied from $1.610^{11}$ to $1.210^{12}$ 
photons $/$ pulse $/ \mathrm{cm}^{2}$. The TRPL decays are fast, barely longer than our instrument response function full width at half maximum (600 ps).

In common MPL/MEL experiments [2,3,10], the PL modulated intensity is expressed as a complex fraction $\mathrm{I}_{\mathrm{MPL}} \propto \frac{\widehat{\mathrm{G}} \tau_{\text {eff }}}{1+2 \pi \mathrm{jf} \tau_{\text {eff }}}$ depending on the modulation frequency $f$, the sinusoidal part of the generation rate $\widehat{G}$ and a single effective lifetime $\tau_{\text {eff }}$. The PL phase would be in this case $\varphi_{\mathrm{MPL}}=$ $-\operatorname{atan}\left(2 \pi f \tau_{\text {eff }}\right)$. This formula is equivalent to a mono-exponential decay in temporal domain and leads to a monotonically decreasing phase with respect to the modulation frequency. However, the fact that the PL phase delay presents a $\mathrm{V}$-shape behavior prevents the use of mono-exponential equivalent model and leads us to use the formulation of Pawlack et al. [21,22]. It expresses a multiexponential-like behavior as a sum of complex fractions in the frequency domain. This would lead in our $V$-shape case to the existence of two "lifetimes" $\tau_{1}$ and $\tau_{2}$, with $I_{M P L} \propto \widehat{G}\left(\frac{\tau_{1}}{1+2 \pi j f \tau_{1}}+\frac{A \tau_{2}}{1+2 \pi j f \tau_{2}}\right)$, and $A$ being a proportionality coefficient between the two contributions. Attempts to fit the MPL curves one by one gave positive results (not presented here), situating $\tau_{1}$ in the $0.5-1.5 \mathrm{~ns}$ range, $\tau_{2}$ between $100-600 \mathrm{~ns}$, and $A$ between $10^{-3}$ and $10^{-4}$, all three values being reduced by the laser intensity. Bi-exponential behavior has already been studied in the temporal domain by Maiberg et al. $[6,23]$ concluding that a shallow trap defect was competing with a fast recombination mechanism. Following this explanation, we introduced in our code one trap center and one SRH recombination center using rate equations for the defects $[6,17]$. We situated in a first approximation the energy position of the SRH center at mid-gap. In the same way as [6], this SRH deep center is supposed to represent the dominant recombination mechanism, which could be situated in the bulk or at the interfaces. We fitted simultaneously the five phase curves matching reasonably well the data. The net doping concentration being not perfectly known; its value was varied in our simulation from $1 \times 10^{16} \mathrm{~cm}^{-3}$ to $8 \times 10^{16} \mathrm{~cm}^{-3}$ without drastic change in the obtained parameters. The other simulation parameters are presented in table 1 . The corresponding shallow trap density was found close to $1-$ $2 \times 10^{17} \mathrm{~cm}^{-3}$, being situated at an energy of $0.19-0.23 \mathrm{eV}$ below the conduction band. The capture cross sections were $3-9 \times 10^{-16} \mathrm{~cm}^{2}$ for electrons and inferior to $10^{-18} \mathrm{~cm}^{2}$ for holes, this center being essentially an electron trap with negligible interaction with the valence band. Note that the value $E_{\text {trap }}=0.2 \mathrm{eV}$ below conduction band has been found by several methods in the past including for example photocapacitance techniques [24], TRPL [6.7], Deep Level Transient Spectroscopy (DLTS) [25]. Our values for the capture cross sections are closer from the DLTS values of Zabierowsky and Edoff [25] than those of Maiberg [6]. The SRH mechanism implies a 0.7-1.3 ns lifetime for electrons and in opposite to [6] a negligible lifetime for holes. Thus, the SRH formula is simplified and the recombination term is finally represented by an injection independent minority carrier lifetime (All the fit parameters are presented in table 2). This is an important result and could imply that the SRH center has a bigger capture cross section for electrons than for holes, or it could also be associated with an interface recombination velocity. Finally, the parameters extracted with HF-MPL allow for fitting the TRPL data. As the TRPL decays are extremely fast, an interpretation was not possible without the help of HF-MPL. In this particular case, the MPL was able to provide more information than the TRPL

\section{2 results with CdS covering}

The results for the CdS covered sample were presented in Figure 3. The light power vas varied from 13 to 6000 suns for HF-MPL and from $1.6 \times 10^{10}$ photon/pulse $/ \mathrm{cm}^{2}$ to $4.9 \times 10^{11}$ photons $/$ pulse $/ \mathrm{cm} 2$ for the TRPL. The fact that the MPL was measurable under lower illumination level ( 13 suns against 130 for the as-grown sample), as well as the increase of the TRPL decay time implies a strong reduction of the recombination mechanisms, i.e. that the CdS is passivating the CIGS absorber. Furthermore, the 
behavior of HF-MPLdynamics as well as TRPL decays are completely different from those of the asgrown sample. The phase curves present monotonic shapes corresponding to arctan function. The deduced time constant is decreasing when injection increases. According to our simulations and supposing a low CdS doping, that could correspond to the presence on a unique really shallow volume trap ( $0.10 \mathrm{meV}$ below conduction band). However, this explanation is not compatible with the simulations of TRPL decays in the same conditions. TRPL decays exhibit strong curvatures in semilogarithmic scale, which could be attributed to bimolecular recombination at the higher injection levels or charge separation due to the junction electric field. In order to obtain a sufficient field effect, the CdS doping density had to be set to $10^{18} \mathrm{~cm}^{-3}$, leading to an increase of the decays curvatures. The possible effect of this field on the trap parameters was taken into account by introducing the trap assisted tunneling formula $[19,25]$, al so providing a supplementary curvature. Then, supposing that the observed trap was the result of an interface modification by $\mathrm{CdS}$, we limited its position to the vicinity of the interface, in the first $250 \mathrm{~nm}$ of the absorber. All these hypotheses were necessary to fit both TRPL and HF-MPL data. The defect energy level is found at 170 meV below the conduction band, the trap density is $5-10 \times 10^{17} \mathrm{~cm}^{-3}$ and the capture cross section $2-5 \times 10^{-15} \mathrm{~cm}^{2}$ for electrons and $8-9 \times 10^{-17} \mathrm{~cm}^{2}$ for holes. In this case, TRPL provided more information, but MPL was helpful for validating the hypotheses.

\subsection{Defects location}

As we mentioned to fit as-grown sample data we have introduced two bulk mechanisms. It is worth noting that having a single bulk trap level plus a surface recombination does not allow us to fit the data. We conclude that these mechanisms are either associated to volume or grain boundaries defects, i.e. three dimensional mechanisms.

In an opposite way, we had to build a different model for the CdS covered sample with recombination traps located at the vicinity of the interface. The fast volume recombination mechanism as well as the volume trap center present in the as grown sample disappeared with CdS deposition. This could be an argument for locating this two centers at the grain boundaries of the asgrown absorber. Indeed, CdS bath deposition has been found to deeply modify the recombination at grain boundaries [26]. In the same way for CdS covered sample, we tried to increase the thickness of the defect layer without success, confirming the interfacial nature of these defect, located at the $\mathrm{CIGS} / \mathrm{CdS}$ interface. This is in accordance with recent depth resolved DLTS experiments [27].

\section{Conclusion}

High Frequency Modulated Photoluminescence experiments were conducted on a CIGS sample either as grown, or with a CdS layer. The proposed setup allows recording fast modulated data while keeping high detection sensitivity. TRPL decays were also acquired and data from both experimental setups were fitted using one dimensional time-dependent drift diffusion model. The as-grown sample was found to contain a shallow volume trap center with $0.2 \mathrm{eV}$ energetic position below the conduction band and a fast recombination mechanism. After CdS deposition, HF-MPL as well as TRPL curves changed drastically. This is attributed to an interface defect situated at $0.17 \mathrm{eV}$ below the conduction band but with slightly different capture cross sections and density. In order to explain the change from volume to interfacial trapping after CdS covering and the disappearance of the fast recombination mechanism, the authors suggest than grain boundaries were passivated by $\mathrm{CdS}$. A proof of concept has been made than the HF-MPL provide complementary information to TRPL and is suitable for defect tracking in thin film absorbers. 


\section{Acknowledgment}

This work was supported by the French government in the frame or the program of investments for the future (Programme d'Investissement d'Avenir ANR-IEED-002-01).

We gratefully acknowledge Sofia Gaiaschi from Horiba France for performing glow discharge optical emission spectroscopy.

[1] K.B. Choi, C.S.P. Teena, J.W. Ho, J. Wang, J. Lin, A.A. Bettiol, A.G. Aberle, J. Wong, Modulated Photoluminescence Lifetime Measurement of Bifacial Solar Cells, IEEE J. Photovolt. 7 (2017) 1687-1692. doi:10.1109/JPHOTOV.2017.2733163.

[2] R. Brüggemann, S. Reynolds, Modulated photoluminescence studies for lifetime determination in amorphous-silicon passivated crystalline-silicon wafers, J. Non-Cryst. Solids. 352 (2006) 18881891. doi:10.1016/j.jnoncrysol.2005.11.092.

[3] W. Favre, J.-P. Kleider, D. Muñoz, S. Martin-de-Nicolás, P.-J. Ribeyron, Spatially resolved lifetime measurements of silicon heterojunctions from the modulated photoluminescence technique, Phys. Status Solidi C. 8 (2011) 775-778. doi:10.1002/pssc.201000286.

[4] M. Maiberg, R. Scheer, Theoretical study of time-resolved luminescence in semiconductors. I. Decay from the steady state, J. Appl. Phys. 116 (2014) 123710. doi:10.1063/1.4896483.

[5] M. Maiberg, R. Scheer, Theoretical study of time-resolved luminescence in semiconductors. II. Pulsed excitation, J. Appl. Phys. 116 (2014) 123711. doi:10.1063/1.4896484.

[6] M. Maiberg, T. Hölscher, S. Zahedi-Azad, R. Scheer, Theoretical study of time-resolved luminescence in semiconductors. III. Trap states in the band gap, J. Appl. Phys. 118 (2015) 105701. doi:10.1063/1.4929877.

[7] M. Maiberg, T. Hölscher, E. Jarzembowski, S. Hartnauer, S. Zahedi-Azad, W. Fränzel, R. Scheer, Verification of minority carrier traps in $\mathrm{Cu}(\mathrm{In}, \mathrm{Ga}) \mathrm{Se}_{2}$ and $\mathrm{Cu}_{2} \mathrm{ZnSnSe}_{4}$ by means of time-resolved photoluminescence, Thin Solid Films. 633 (2017) 208-212. doi:10.1016/j.tsf.2017.02.002.

[8] D. Kuciauskas, J.V. Li, M.A. Contreras, J. Pankow, P. Dippo, M. Young, L.M. Mansfield, R. Noufi, D. Levi, Charge carrier dynamics and recombination in graded band gap Culn ${ }_{1-x} \mathrm{Ga}_{\times} \mathrm{Se}_{2}$ polycrystalline thin-film photovoltaic solar cell absorbers, J. Appl. Phys. 114 (2013) 154505. doi:10.1063/1.4825211.

[9] L. Lombez, M. Soro, A. Delamarre, N. Naghavi, N. Barreau, D. Lincot, J. -F. Guillemoles, Revisiting the interpretation of biased luminescence: Effects on $\mathrm{Cu}(\mathrm{In}, \mathrm{Ga}) \mathrm{Se} 2$ photovoltaic heterostructures, J. Appl. Phys. 116 (2014) 064504. doi:10.1063/1.4891525.

[10] S. Khatavkar, K. Muniappan, C.V. Kannan, V. Kumar, K.L. Narsimhan, P.R. Nair, J.M. Vasi, M.A. Contreras, M.F.A.M. van Hest, B.M. Arora, Measurement of Relaxation Time of Excess Carriers in Si and CIGS Solar Cells by Modulated Electroluminescence Technique, Phys. Status Solidi A. 215 (2018) 1700267. doi:10.1002/pssa.201700267.

[11] M. Xu, Photoluminescence Techniques for the Characterization of Photovoltaic Interfaces, PhD Thesis, Paris Saclay, 2016. http://www.theses.fr/2016SACLS053.

[12] J.W. Eaton, D. Bateman, S. Hauberg, R. Wehbring, GNU Octave version 4.2.1 manual: a high-level $\begin{array}{llll}\text { interactive language for numerical computations, } 2017 . & \end{array}$ https://www.gnu.org/software/octave/doc/v4.2.1/.

[13] P. Farrell, N. Rotundo, D. Hai Doan, M. Kantner, J. Fuhrmann, T. Koprucki, Numerical methods for drift-diffusion models, Weierstraß-Institut für Angewandte Analysis und Stochastik, 2016. http://www.wias-berlin.de/preprint/2263/wias_preprints_2263.pdf.

[14] P.E. Van Keken, D.A. Yuen, L.R. Petzold, DASPK: A new high order and adaptive time-integration technique with applications to mantle convection with strongly temperature-and pressuredependent rheology, Geophys. Astrophys. Fluid Dyn. 80 (1995) 57-74. doi:10.1080/03091929508229763. 
[15] A.C. Hindmarsh, P.N. Brown, K.E. Grant, S.L. Lee, R. Serban, D.E. Shumaker, C.S. Woodward, SUNDIALS: Suite of nonlinear and differential/algebraic equation solvers, ACM Trans. Math. Softw. TOMS. 31 (2005) 363-396.

[16] C. de Falco, R. Sacco, M. Verri, Analytical and numerical study of photocurrent transients in organic polymer solar cells, Comput. Methods Appl. Mech. Eng. 199 (2010) 1722-1732. doi:10.1016/j.cma.2010.01.018.

[17] B. Berger, N. Schüler, S. Anger, B. Gründig-Wendrock, J.R. Niklas, K. Dornich, Contactless electrical defect characterization in semiconductors by microwave detected photo induced current transient spectroscopy (MD-PICTS) and microwave detected photoconductivity (MDP), Phys. Status Solidi A. 208 (2011) 769-776. doi:10.1002/pssa.201083994.

[18] K. Yang, J.R. East, G.I. Haddad, Numerical modeling of abrupt heterojunctions using a thermionicfield emission boundary condition, Solid-State Electron. 36 (1993) 321-330. doi:10.1016/00381101(93)90083-3.

[19] J. Furlan, Z. Gorup, F. Smole, M. Topic, MODELING TUNNELING-ASSISTED GENERATIONRECOMBINATION RATE IN SPACE-CHARGE REGION OF PN A-SI:H JUNCTION, J Model. Simul. Microsyst. 1 (1999) 109-114.

[20] G. Vincent, A. Chantre, D. Bois, Electric field effect on the thermal emission of traps in semiconductor junctions, J. Appl. Phys. 50 (1979) 5484. doi:10.1063/1.326601.

[21] M. Pawlak, K. Strzałkowski, Identification of the photoluminescence response in the frequency domain modulated infrared radiometry signal of ZnTe:Cr bulk crystal, Infrared Phys. Technol. 78 (2016) 190-194. doi:10.1016/j.infrared.2016.08.005.

[22] M. Pawlak, Comments on the paper: M. Pawlak, K. Strzakowski, Identification of the photoluminescence response in the frequency domain modulated infrared radiometry signal of ZnTe:Cr bulk crystal, Infrared Phys. Technol. 78 (2016) 190-194, Infrared Phys. Technol. 85 (2017) 502-503. doi:10.1016/j.infrared.2017.05.016.

[23] M. Maiberg, T. Hölscher, S. Zahedi-Azad, W. Fränzel, R. Scheer, Investigation of long lifetimes in $\mathrm{Cu}(\mathrm{In}, \mathrm{Ga}) \mathrm{Se}_{2}$ by time-resolved photoluminescence, Appl. Phys. Lett. 107 (2015) 122104. doi:10.1063/1.4931632.

[24] M. Igalson, A. Urbaniak, Defect states in the GIGS solar cells by photocapacitance and deep level optical spectroscopy, Bull. Pol. Acad. Sci. Tech. Sci. 53 (2005) 157-161.

[25] P. Zabierowski, M. Edoff, Laplace-DLTS analysis of the minority carrier traps in the $\mathrm{Cu}(\mathrm{In}, \mathrm{Ga}) \mathrm{Se}_{2}$ based solar cells, Thin Solid Films. 480-481 (2005) 301-306. doi:10.1016/j.tsf.2004.11.005.

[26] C. Lei, M. Duch, I.M. Robertson, A. Rockett, Effects of solution-grown CdS on Cu(InGa)Se2 grain boundaries, J. Appl. Phys. 108 (2010) 114908. doi:10.1063/1.3512966.

[27] S. Heo, J. Chung, H.-I. Lee, J. Lee, J.-B. Park, E. Cho, K. Kim, S.H. Kim, G.S. Park, D. Lee, J. Lee, J. Nam, J. Yang, D. Lee, H.Y. Cho, H.J. Kang, P.-H. Choi, B.-D. Choi, Defect visualization of $\mathrm{Cu}(\operatorname{InGa})(\mathrm{SeS}) 2$ thin films using DLTS measurement, Sci. Rep. 6 (2016) 30554. doi:10.1038/srep30554. 
CIGS thickness $d$

CIGS

CdS

CIGS doping $N_{a}$

$2.3 \mu \mathrm{m}$

$40 \mathrm{~nm}$

Relative permittivity $\varepsilon_{r}$

Electron affinity

Electron mobility $\mu_{n}$

Hole mobility $\mu_{p}$

Conduction band density of state $N_{c}$

Valence band density of state $N_{v}$

Bandgap Energy $E_{g}$

Auger coefficients $C_{n}, C_{p}$

Thermal velocities $v_{n}, v_{p}$

Light absorption coefficient at $532 \mathrm{~nm}$

Light absorption coefficient at $640 \mathrm{~nm}$

Radiative recombination coefficient

$\begin{array}{ll}1-8 \times 10^{16} \mathrm{~cm}^{-3} & 1-8 \times 10^{16} \mathrm{~cm}^{-3} \\ 13.6 & 10 \\ 4.1 \mathrm{eV} & 3.8 \mathrm{eV} \\ 100 \mathrm{~cm}^{2} \cdot \mathrm{V}^{-1} \cdot \mathrm{s}^{-1} & 390 \mathrm{~cm}^{2} \cdot \mathrm{V}^{-1} \cdot \mathrm{s}^{-1} \\ 50 \mathrm{~cm}^{2} \mathrm{~V}^{-1} \mathrm{~s}^{-1} & 50 \mathrm{~cm}^{2} \mathrm{~V}^{-1} \mathrm{~s}^{-1} \\ 6.7 \times 10^{17} \mathrm{~cm}^{-3} & 10^{18} \mathrm{~cm}^{-3} \\ & \\ 1.7 \times 10^{19} \mathrm{~cm}^{-3} & 2 \times 10^{19} \mathrm{~cm}^{-3} \\ 1.15 \mathrm{eV} & 2.4 \mathrm{eV} \\ 10^{-28} \mathrm{~cm}^{-6} \mathrm{s.}^{-1} & \text { neglected } \\ 10^{7} \mathrm{~cm} \cdot \mathrm{s}^{-1} & 10^{7} \mathrm{~cm} \cdot \mathrm{s}^{-1} \\ 1.45 \times 10^{5} \mathrm{~cm}^{-1} & \text { neglected } \\ 8.5 \times 10^{4} \mathrm{~cm}^{-1} & \\ 8 \times 10^{-11} \mathrm{~cm}^{-3} \cdot \mathrm{s}^{-1} & \text { neglected } \\ & \end{array}$




\begin{tabular}{lc|cccc}
\multicolumn{2}{c|}{ SRH Lifetimes } & \multicolumn{4}{|c}{ Trap parameter } \\
\hline Electron & Hole & $\begin{array}{c}\text { Electron capture cross } \\
\text { section }\end{array}$ & $\begin{array}{c}\text { Hole capture cross } \\
\text { section }\end{array}$ & Energy below CB & Trap density \\
$0.7-1.3 \mathrm{~ns}$ & negligible & $3-9 \times 10^{-16} \mathrm{~cm}^{2}$ & $<10^{-18} \mathrm{~cm}^{2}$ & $0.18-0.23 \mathrm{eV}$ & $1-2 \times 10^{17} \mathrm{~cm}^{-3}$
\end{tabular}


Fig. 1. a) Schematic of the setup. Laser is either a $532 \mathrm{~nm}$ intensity modulated laser or a $640 \mathrm{~nm}$ pulsed laser ( 80 ps pulse duration at $10 \mathrm{MHz}$ repetition rate). b) Example of modulated PL signal measured with the TCSPC: direct measurement of the laser light (black), PL signal of a glass/Mo/CIGS/CdS sample (red) presenting a phase shift (delay) with respects to the laser light modulation, Instrument response function is shown in green.

Fig. 2. Left: phases of the modulated PL signals versus modulation frequency measured on the asgrown CIGS sample (crosses) at several illumination powers and the corresponding fits by numerical simulations (lines). Laser power increases from black $\left(1.310^{19}\right.$ photons $\left./ \mathrm{cm}^{2} / \mathrm{s}\right)$ to green, blue, red, cyan with a factor $\sqrt{10}$ at each step. Right: TRPL signals measured on the same sample at several illumination powers (circles) and their fits (lines). Laser power increases from purple (1.6 $10^{11}$ photons/pulse $/ \mathrm{cm}^{2}$ to yellow $(X 2)$, red (X 5), blue (X 8).

Table 1. Parameters used in our simulations

Table 2. Parameters extracted from data fit for the as-grown sample

Fig. 3. Left: phases of the modulated PL signals versus modulation frequency measured on the CdS covered CIGS sample (crosses) at several illumination powers and the corresponding fits by numerical simulations (lines). Laser power increases from black $\left(1.310^{18}\right.$ photons $\left./ \mathrm{cm}^{2} / \mathrm{s}\right)$ to, green, blue, red, cyan, magenta, yellow with a factor $\sqrt{10}$ at each step. Right: TRPL signals measured on the same sample at several illumination powers (circles) and the corresponding fits (lines). Laser power increases from green $\left(1.6 \times 10^{10}\right.$ photon/pulse $\left./ \mathrm{cm}^{2}\right)$ to purple $(X 3)$, orange $(X 10)$, red $(X 30)$. Arrows indicate the increase of illumination. 
a)

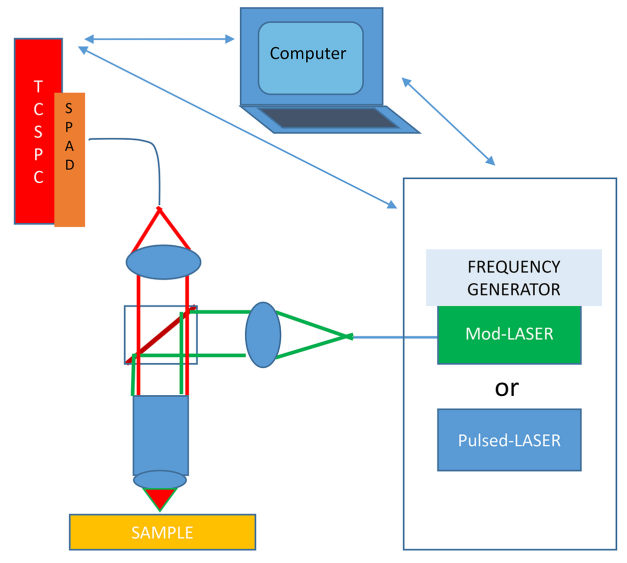

b)

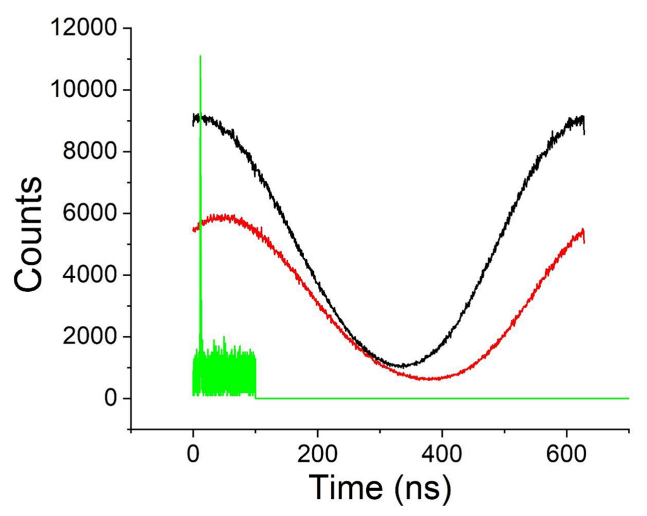

Figure 1 

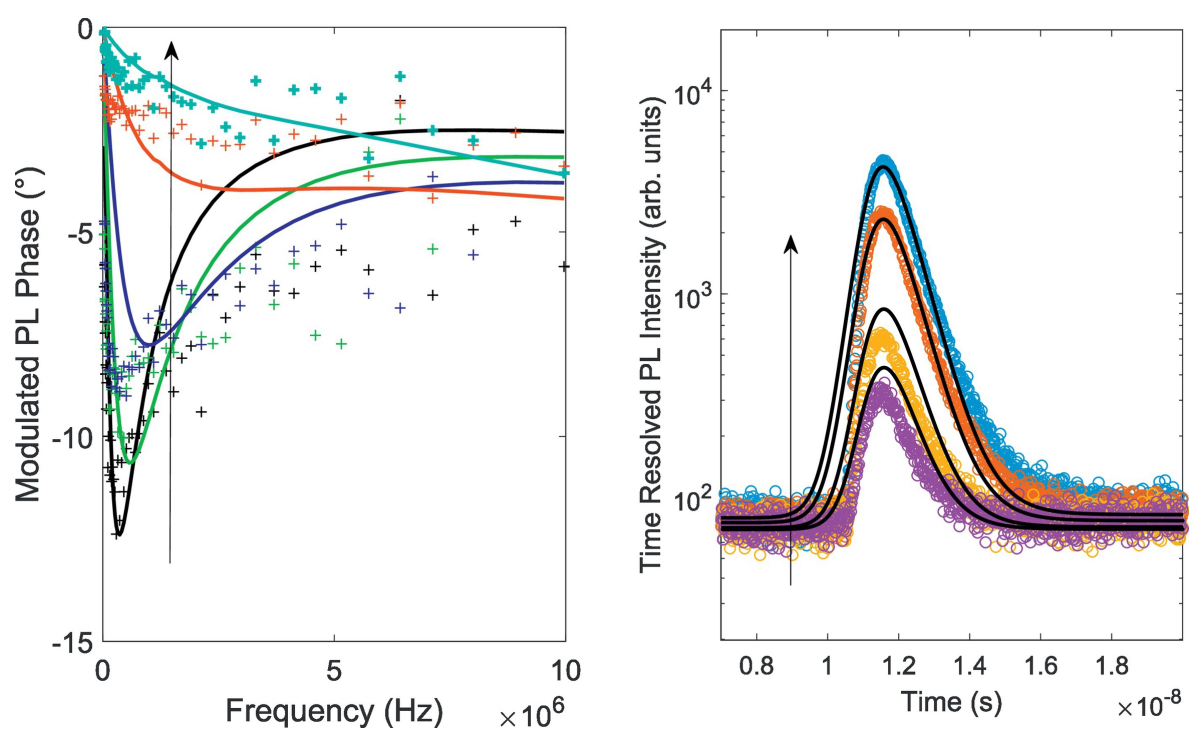

Figure 2 

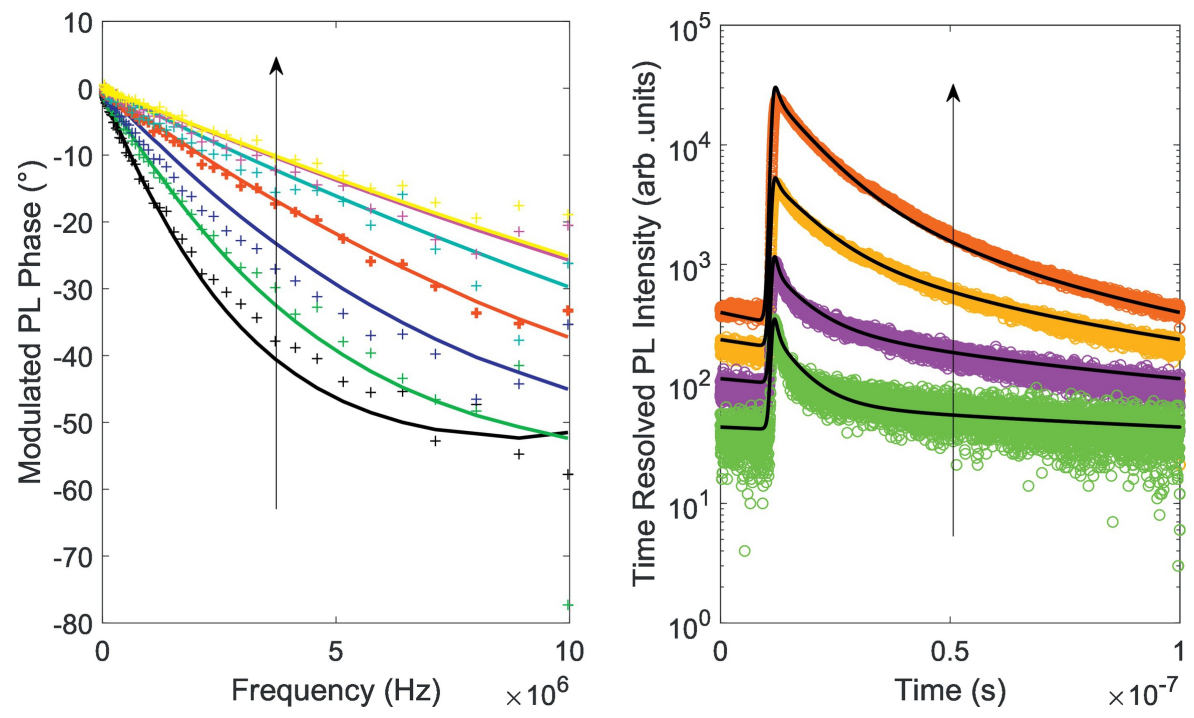

Figure 3 